\title{
Ultra-High-Q Wedge Resonators with Precise FSR control
}

\author{
Hansuek Lee, Tong Chen, Jiang Li, Ki Youl Yang, Oskar Painter, and Kerry Vahala \\ Thomas J. Watson, Sr., Laboratory of Applied Physics, California Institute of Technology, \\ 1200 E. California Boulevard, Pasadena, CA 91125, USA \\ vahala@caltech.edu
}

\begin{abstract}
Optical resonators with Q values of nearly 1 billion are demonstrated, the highest for any chip-based devices. Fabrication uses only standard semiconductor processes, enabling precise size control and access to microwave-rate free-spectral-range operation. OCIS codes: (140.4780) Optical resonators; (140.3945) Microcavities; (230.0230) Optical devices.
\end{abstract}

\section{Introduction}

High-Q performance in microcavities relies critically upon use of low absorption dielectrics and creation of very smooth (low scattering) interfaces. For chip-compatible devices, silica has by far the lowest intrinsic material loss [1]. Microtoroid resonators combine this low material loss with a reflow technique in which surface tension is used to smooth lithographic and etch-related blemishes [2]. At the same time, reflow smoothing makes it very challenging to fabricate larger diameter UHQ resonators and likewise to leverage the full range of integration tools and devices available on silicon. The devices reported here attain ultra-high-Q performance using only conventional semiconductor processing methods on a silicon wafer. Moreover, the best Q performance occurs for diameters greater than 500 microns, a size range that is difficult to access for microtoroids on account of the limitations of the reflow process. Microcombs will benefit from such a combination of UHQ and larger diameter resonators (microwave-rate free-spectral-range) to create combs that are both efficient in turn-on power and that can be selfreferenced [3]. Moreover, integrated reference cavities and ring gyroscopes are two other applications that can benefit from larger ( $1-50 \mathrm{~mm}$ diameter) UHQ resonators. Fabrication control of the free-spectral range (FSR) to 1 : 20,000 is also demonstrated here, opening the possibility of precision repetition rate control in microcombs or precision spectral placement of modes in certain nonlinear oscillators $[4,5]$.

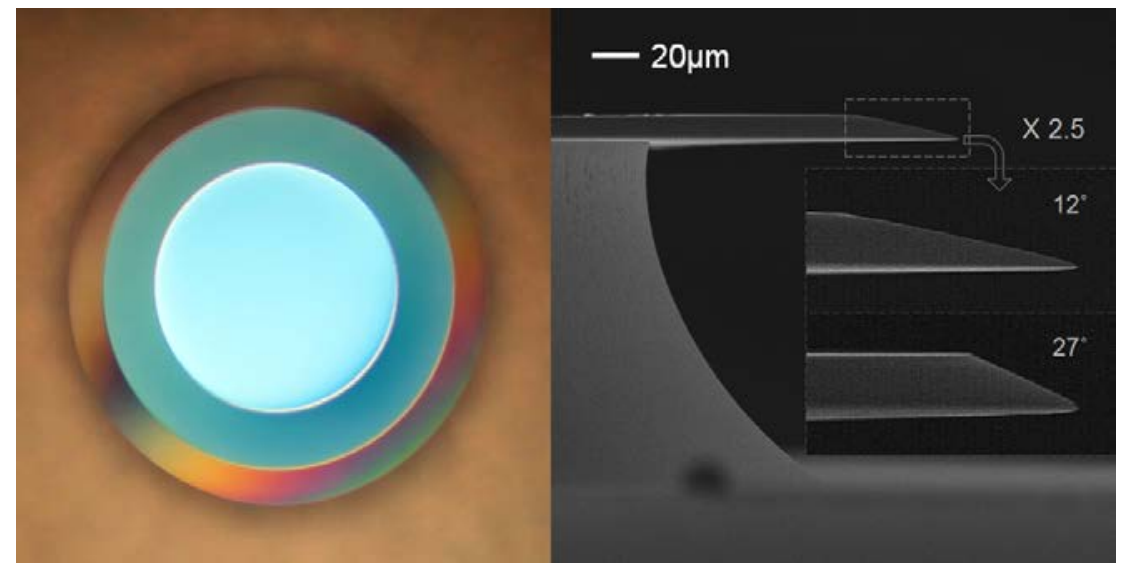

Figure 1. (Left) An optical micrograph shows a top view of a $1 \mathrm{~mm}$ diameter wedge resonator. (Right) A scanning electron micrograph shows the side-view of a resonator. The insets give slightly magnified micrographs of resonators in which the wedge angle is 12 degrees (upper inset) and 27 degrees (lower inset).

\section{Resonator design and fabrication}

The resonator described here is motivated by earlier work in which a resonator having a distinctive wedge-shaped cross section was described [6]. In that work, the mode was isolated from the lithographic blemishes near the outer rim of the resonator by using a shallow wedge angle, resulting in $\mathrm{Q}$ factors as high as 50 million. In the current work, we have boosted the optical Q by about $20 \mathrm{X}$ beyond these earlier results through a combination of process improvements. The fabrication process begins with thermal oxide on silicon, followed by photo-lithography and oxide etching with buffered hydrofluoric acid. The oxide disk structures function as an etch mask for an isotropic $\mathrm{XeF}_{2}$ dry etch of the silicon. The final device is shown in figure 1 . The wedge angle can be controlled in the range from $10^{\circ}$ to $30^{\circ}$ as shown in the inset of figure 1 through adjustment of the photoresist adhesion using commercially available adhesion promoters. 


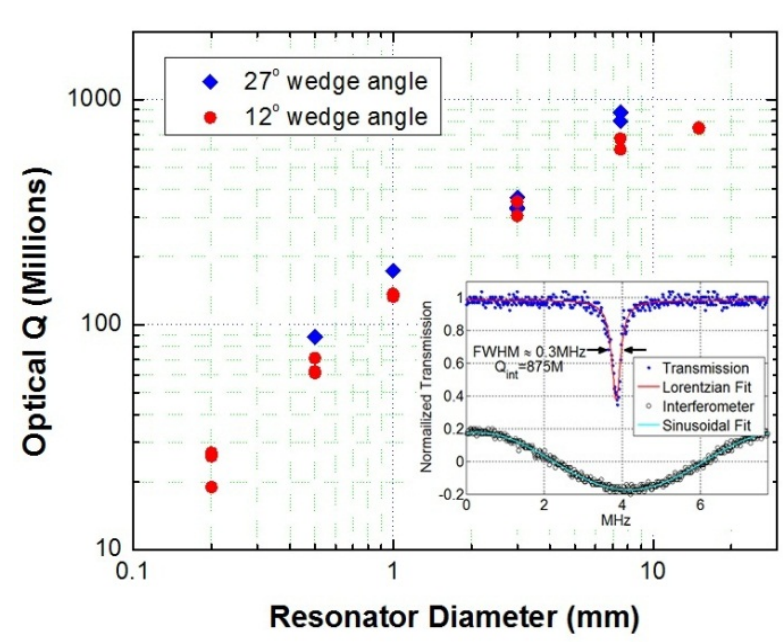

Figure 2. The measured $\mathrm{Q}$ factor plotted versus resonator diameter. $10 \mu \mathrm{m}$ oxide thickness is used for both of the wedge angles shown $\left(12^{\circ}, 27^{\circ}\right)$. The inset shows a spectral scan for the case of a record Q factor of 875 million. The sinusoidal curve accompanying the spectrum is a calibration scan performed using a fiber interferometer.

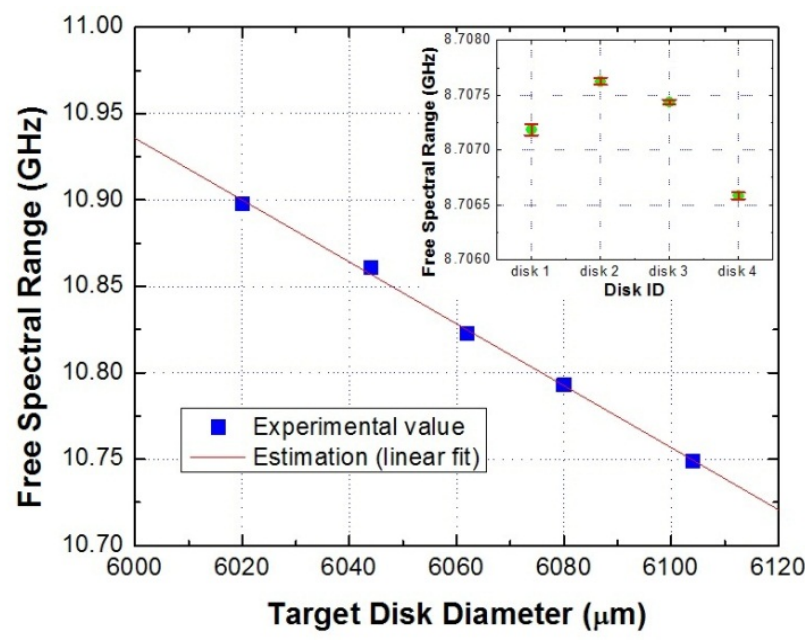

Figure 3. The measured free spectral range (FSR) versus the target design-value resonator diameter on a lithographic mask. The plot shows one device at each size and five different sizes. The rms variance is $2.4 \mathrm{MHz}$. The inset shows the FSR data measured on four devices having the same target FSR. An improved variance of $0.45 \mathrm{MHz}$ is obtained.

\section{Quality factor and Control of FSR (Free Spectral Range)}

Intrinsic Q factor was obtained by measuring spectral line shapes using a tunable external cavity semiconductor laser. Coupling to the resonators relied upon an optical taper [7, 8]. For accurate calibration of the laser scan, a portion of the laser output was also monitored using a calibrated Mach-Zehnder interferometer having a free spectral range of 7.75 MHz. Figure 2 shows Q factor measurements for various resonator sizes and for two different wedge angles, $12^{\circ}$ and $27^{\circ}$. The inset in figure 2 shows a spectral scan obtained on a device having a record Q factor of 875 million. The $\mathrm{Q}$ factor for these new resonators is not only higher in an absolute sense than what has been possible with microtoroids, but it also accesses an important regime of resonator FSR that has not been possible using microtoroids. This range has become increasingly significant in applications like microcombs where self-referencing is important [3].

The ability to lithographically define ultra-high-Q resonators as opposed to relying upon the reflow process enables a multi-order-of-magnitude improvement in precision control of resonator diameter and FSR. As a preliminary test of the practical limits of FSR control, two studies were conducted. In the first, a series of resonator diameters were set in a CAD file used to create a photo mask. A plot of the measured FSR (fundamental mode) versus CAD file target diameter is provided in figure 3 (main panel). The variance from ideal linear behavior is $2.4 \mathrm{MHz}$, giving a relative variance of better than $1: 4,500$ (FSR $11 \mathrm{GHz}$ ). The inset to figure 3 shows that for separate devices having the same target CAD file diameter, the variance is further improved to a value of $0.45 \mathrm{MHz}$ or $1: 20,000(\mathrm{FSR} \approx 8.7 \mathrm{GHz})$. This level of control makes it possible to precisely tune microcomb repetition rate and also place the FSR at the Brillouin shift in stimulated Brillouin microlasers. These applications will be described elsewhere.

\section{References}

[1] K. J. Vahala, "Optical Microcavities," Nautre 424, 839-846 (2003).

[2] D. K. Armani, T. J. Kippenberg, S. M. Spillane \& K. J. Vahala, "Ultra-high-Q toroid microcavity on a chip.” Nature 421, 925-929 (2003).

[3] T. J. Kippenberg, R. Holzwarth \& S. A. Diddams, "Microresonator-based optical frequency combs." Science 332, 555-559 (2011).

[4] M. Tomes, \& T. Carmon, "Photonic micro-electromechanical systems vibrating at X-band (11-GHz) rates." Phys. Rev. Lett. 102,113601 (2009).

[5] I. S. Grudinin, N. Yu \& L. Maleki, "Brillouin lasing with a CaF2 whispering gallery mode resonator.” Phys. Rev. Lett. 102, 043902 (2009).

[6] T. J. Kippenberg, J. Kalkman, A. Polman \& K. J. Vahala, "Demonstration of an erbium-doped microdisk laser on a silicon chip." Phys. Rev. A $74,051802(2006)$.

[7] M. Cai, O. J. Painter \& K. J. Vahala, "Observation of critical coupling in a fiber taper to silica-microsphere whispering gallery mode system. Phys. Rev. Lett. 74, 051802 (2006).

[8] S. M. Spillane, T. J. Kippenberg, O. J. Painter \& K. J. Vahala, "Ideality in a fiber-taper-coupled microresonator system for application to cavity quantum electrodynamics.," Phys. Rev. Lett. 91, 043902 (2003). 